\title{
2012 Wilderness E Environmental Medicine Peer Reviewers
}

\begin{abstract}
The editors wish to express their sincere appreciation to the following individuals who faithfully and skillfully performed peer reviews for articles submitted to Wilderness \& Environmental Medicine. WEM continues to have a growing impact on the scientific community. We want to acknowledge the importance of our peer reviewers to the process of ensuring the merit and quality of the manuscripts that we publish. Many of these peer reviewers reviewed multiple papers, and some also serve on the Editorial Board and have Section Editor duties as well, and without them we could not produce this high quality medical journal.

If you are interested in becoming a peer reviewer in your area of expertise for Wilderness \& Environmental Medicine in 2013, please send your CV to Editor-in-Chief Scott E. McIntosh (scott.mcintosh@hsc.utah.edu) and Managing Editor Jonna Barry (jonna@wms.org).
\end{abstract}

Adam Algren, USA

John Anderson, USA

Howard Backer, USA*

Peter Bartsch, Germany

Buddha Basnyat, Nepal*

Benjamin Bassin, USA

Cynthia M. Beall, USA

Claus Behn, Chile

Jesus Benitez, Venezuela

Brad L. Bennett, USA*

Marc Moritz Berger, Germany

Jeffery Bernstein, USA

Aaron R. Billin, USA

Lee Bogle, USA

Mark E. Brauner, USA

Hermann Brugger, Italy

Frank K. Butler, USA

Michael D. Cardwell, USA

Edward Castillo, USA

Michael Caudell, USA

Mario Cavazza, Italy

Nathan P. Charlton, USA

Julie Chase, USA

Kenneth Chiacchia, USA

Kira Christian, USA

Keith Conover, USA

Ben Constance, USA

Dan Danzl, USA

Michael A. Darracq, USA

Christopher B. Davis, USA

Peter Davis, UK

Petar DeNoble, USA

Robert W. Derlet, USA

Steve Donelan, USA*

Jennifer Dow, USA

Ola H. Dunin-Bell, Canada

Bruno Durrer, Switzerland*

*Wilderness \& Environmental Medicine Board Member and/or Section Editor
Mark Edsell, UK

Gretchen Ela, USA

Preston J. Fedor, USA

Peter Fenner, Australia*

Lance Ferguson, USA

William Forgey, USA

Joseph D. Forrester, USA

Luanne Freer, USA*

Nathalie Garcia Russell, USA

Flavio G. Gaudio, USA

Christopher Gee, USA

Giovanni Ghirga, Italy

Gordon Giesbrecht, Canada*

Torrey L. Goodman, USA

Mark W. Greve, USA

Colin K. Grissom, USA*

Cara Haberman, USA

Peter H. Hackett, USA*

Vidal Haddad, Jr., Brazil

Matt Hamonko, USA

N. Stuart Harris, USA

Christopher Hearon, USA

Travis W. Heggie, USA

Carlton Heine, USA

Tamara Hew-Butler, USA

David Hillebrandt, UK

Alastair Hodges, Canada

Martin D. Hoffman, USA

Christopher P. Holstege, USA

Sean Hudson, UK

Angus Hunter, UK

Timothy R. Hurtado, USA

Christopher H.E. Imray, UK

Geoffrey Isbister, Australia

Kenneth V. Iserson, USA

Nicholas S. Kalson, UK

Christopher Kang, USA

Lee Kaplan, USA

Bengt Kayser, Switzerland
Kenneth W. Kizer, USA*

Judith Klein, USA*

Toshio Kobayashi, Japan

Michael Koehle, Canada

Ricky Langley, USA

Mike Langran, UK

Matthew P. Lazio, USA

Fabiola Leon-Velarde, Peru

Gemma Lewis, USA

Cynthia Lewis-Younger, USA

James Lieberman, USA

Louis Ling, USA

Grant S. Lipman, USA

Lanny Littlejohn, USA

Felipe Lorenzo, USA

Santiago Lorenzo, USA

Andrew M. Luks, USA

Martin MacInnis, Canada

Thomas Mader, USA

S. Mahadeva, India

Claude Maillaud, New Caledonia

Tom E. Mallinson, UK

Jayme Mancini, USA

Giorgio Mazzuero, Italy

Daniel McBride, USA

John T. McClain, USA

Marion McDevitt, USA

Timothy McGraw, USA

Manuel Mendoza, USA

Michael G. Millin, USA

Mitchell Mitchell, UK

Chris Moore, USA

Fernando A. Moraga, Chile

Dan Morris, UK

Audry Morrison, UK

Roger B. Mortimer, USA

Andrew Muck, USA

Matthew D. Muller, USA

Stephen Muza, USA 
Arnold G. Nelson, USA

Mária Némethy, USA

Timothy Noakes, South Africa

Robert Norris, USA

John Ogle, USA

Sheryl Olson, USA

Kathy Orloski, USA

Edward Otten, USA*

Peter Paal, Austria

Ryan Paterson, USA

Andre M. Pennardt, USA

Patrick Peters, Luxembourg

Neal W. Pollock, USA*

R. Ponampala, Singapore

Robin Pope, USA

Matiram Pun, Canada

Robert Quinn, USA

Naeem Quraishi, India

Billy Rice, USA

Jean-Paul Richalet, France

Alan J. Richardson, UK
George W. Rodway, USA*

Ian Rogers, Australia*

Anne-Michelle Ruha, USA

James L. Rupert, Canada

Emily Sagalyn, USA

Tod Schimelpfenig, USA

Robert Schoene, USA*

Volker Schöffl, Germany

Mary Beth Scholand, USA

Mark Shenkin, USA

Mariusz Sieminski, Poland

Tatum Simonson, USA

Alok Sinha, USA

JohnEric Smith, USA

Will R. Smith, USA

Cynthia B. Stevens, USA

Cris Stickley, USA

Joshua O. Stream, USA

John B. Sullivan, Jr., USA

Erik R. Swenson, USA

Nathaniel Szewczyk, UK
Geoffrey C. Tabin, USA

Shani Tan, British Columbia

David A. Townes, USA

Maria Urso, USA

Christopher Van Tilburg, USA

Richard Vetter, USA

Gines Viscor, Spain

Scott R. Votey, USA

Dale R. Wagner, USA

John B. Walden, USA

David Warrell, UK

Scott A. Weinstein, USA

Julie Welch, USA

Klaas Westerterp, Netherlands

James A. Wilkerson, III, USA

Alexander D. Wright, UK

Michael Yaron, USA

Mustfa Yildiz, Turkey

Cedric M. Yoshimoto, USA

Ken Zafren, USA*

Gustavo R. Zubieta-Calleja, Bolivia 